\title{
A novel insulator by holographic Q-lattices
}

Yi Ling, ${ }^{a, c}$ Peng Liu ${ }^{a}$ and Jian-Pin $\mathbf{W} \mathbf{u}^{b, c}$

${ }^{a}$ Institute of High Energy Physics, Chinese Academy of Sciences, Beijing 100049, China

${ }^{b}$ Institute of Gravitation and Cosmology, Department of Physics, School of Mathematics and Physics, Bohai University, Jinzhou 121013, China

${ }^{c}$ State Key Laboratory of Theoretical Physics, Institute of Theoretical Physics, Chinese Academy of Sciences, Beijing 100190, China

E-mail: lingy@ihep.ac.cn, liup51@ihep.ac.cn, jianpinwu@mail.bnu.edu.cn

ABSTRACT: We construct a bulk geometry with Q-lattice structure, which is implemented by two gauge fields and a coupling between the lattice and the Maxwell field. This gravity dual model can describe a novel insulator which exhibits some key features analogous to Mott insulator. In particular, a hard gap in insulating phase as well as vanishing DC conductivity can be simultaneously achieved. In addition, we discuss the non-Drude behavior of the optical conductivity in low frequency region in insulating phase, which exhibits some novel characteristics different from ordinary Mott insulator.

KEYwORDS: Holography and condensed matter physics (AdS/CMT), Gauge-gravity correspondence, AdS-CFT Correspondence

ARXIV EPRINT: 1510.05456 


\section{Contents}

1 Introduction 1

2 Mott-like insulating phase 2

$\begin{array}{llr}3 & \text { Discussion } & 8\end{array}$

\section{Introduction}

Metal-insulator transition, which exhibits very appealing and peculiar properties in the electronic conductivity, is a fundamental issue in condensed matter physics [1-4]. In particular, Mott insulator is a typical many-body system in which the transport of electrons is inhibited not due to the full filled band but rather to strong local electronic correlations [5]. For such strongly correlated system, the conventional methods are ineffective and the basic principle behind them is fuzzy. AdS/CFT correspondence provides a powerful tool and new paradigm for understanding strongly correlated systems [6-8]. In holographic approach various novel localization mechanisms have been implemented with the lattice deformation in bulk geometry, which is usually solvable such that it provides an intuitive geometric scenario from gravity side for the dual system with strongly correlated electrons. When the operator breaking the translational symmetry becomes relevant in the IR region, a metal-insulator transition will happen in the dual field theory [9-15]. Different lattice structure results in different localization mechanism such that we have a diverse landscape of insulating phase in a holographic scenario, for instance, Peirels insulator [13], polaron-localization insulating phase [15] or other novel insulating phases exhibiting peculiar characteristics [9-12, 14], some of which are similar to those found in condensed matter.

In this paper, we will construct a holographic model which is dual to a novel insulator that shares some important characteristics of Mott insulator. In this route the pioneer work on the formation of hard gap has appeared in $[16,17]$. In [16], the momentum relaxation is introduced by axion field $\chi_{i}=k x_{i}$ in some gapped geometries [21-23] such that a hard gap in optical conductivity and an insulating ground state can be obtained. But the thought experiment originally proposed by Mott is not transparent in this scenario due to the absence of periodic lattice structure. In Mott thought experiment, when the repulsive force between two electrons is taken into account, a metal could transit into an insulator with the increase of the lattice constant, simply because the hoping ability of electrons decays. In our recent work [17], we introduce a coupling between the lattice and the electromagnetic field over a Q-lattice background which exhibits a manifest periodic structure [11]. We find our model can not only implement the Mott thought experiment explicitly, but also exhibit a hard gap evidently in optical conductivity when the coupling parameter is relatively large. However, in this framework we find the dual system always 
stays in a novel metallic phase in zero temperature limit whenever a hard gap is formed. We conjecture it is a doped system where umklapp scattering is frozen at zero temperature. As a result, a ground state corresponding to a Mott insulating phase at zero temperature is not achieved in [17]. Based on our previous work, in this paper we intend to report a substantial progress to [17]. We will introduce a gravity dual model with two U(1) gauge fields. This two-gauge formalism has been introduced into holographic models for a long time, with a long list of publications, for instance, in $[9,13,18,19]$. It originally comes from the top-down construction of holography based on the low energy limit of superstring or $\mathrm{M}$ theory $[18,20]$. In our current work, the motivation of introducing an additional gauge field is to generate enough deformation of the IR geometry so as to be dual to an insulating phase in zero temperature limit. In our previous work [17], the coupling term between the lattice and the electromagnetic field plays a key role in generating a hard gap in insulating phase. However, at the same time this coupling term will weaken the influence of the electromagnetic field to the background such that the IR deformation due to lattices will becomes weak as well. To solve this difficulty we intend to consider the two-gauge formalism in this paper. We will demonstrate that in the presence of the second gauge field the Q-lattice will strongly deform the IR geometry even when the coupling term becomes strong such that an insulating ground state with hard gap can be obtained in zero temperature limit. In particular, such kind of ground states has vanishing DC conductivity when the coupling parameter is large enough. Some novel features of the model such as the non-Drude behavior will be briefly addressed as well.

\section{Mott-like insulating phase}

We propose the following action as the starting point of our current model

$$
S=\frac{1}{2 \kappa^{2}} \int d^{4} x \sqrt{-g}\left[R+\frac{6}{L^{2}}-|\nabla \Phi|^{2}-\frac{m^{2}}{L^{2}}|\Phi|^{2}-\frac{1}{4} F^{2}-\frac{Z(\Phi)}{4} G^{2}\right],
$$

where $L$ is the AdS radius and $F=d A, G=d B$ are curvatures of two U(1) gauge fields $A$ and $B$, respectively. We will treat $B$ field as the Maxwell field and will concentrate on its transport properties through this paper. The coupling term $Z(\Phi)$ is introduced to describe the interaction between the Q-lattice $\Phi$ and the Maxwell field $B$ in bulk geometry. Specifically, we will set this coupling as $Z(\Phi)=\left(1-\beta|\Phi|^{2}\right)^{2}$ with $\beta$ being positive. It is worthwhile to notice that $Z(\Phi)$ is positive definite such that the stability of system is guaranteed. Previously, a similar coupling has been introduced in a simple holographic model without the breaking of translational symmetry in [24].

Consider the following ansatz for the background fields

$$
\begin{aligned}
d s^{2} & =\frac{1}{z^{2}}\left[-(1-z) p(z) U d t^{2}+\frac{d z^{2}}{(1-z) p(z) U}+V_{1} d x^{2}+V_{2} d y^{2}\right], \\
A & =\mu(1-z) a d t \\
B & =\mu(1-z) b d t \\
\Phi & =e^{i \hat{k} x} z^{3-\Delta} \phi(z),
\end{aligned}
$$


where $p(z)=1+z+z^{2}-\mu^{2} z^{3} / 4$ and scaling dimension of the scalar field $\Delta=3 / 2 \pm$ $\left(9 / 4+m^{2}\right)^{1 / 2}$. For convenience, we have fixed the radius of $\operatorname{AdS} L=1$. All the functions $U, V_{1}, V_{2}, a, b$ and $\phi$ depend on the radial coordinate $z$ only. Through this paper we set $m^{2}=-2$ so that $\Delta=2$. The background solutions to the equations of motions from (2.1) are obtained with full backreaction. We set the boundary conditions $a(0)=1$ and let $b_{0}=b(0)$ be a tunable parameter. The temperature of the dual system is given by

$$
\hat{T}=\frac{\left(12-\mu^{2}\right) \mathrm{U}(1)}{16 \pi} .
$$

Obviously, as $a(z)=1, b(z)=0$ and $\phi(z)=0$ the background solution is nothing but the standard RN-AdS black hole. For a given coupling constant $\beta$, each electrically charged black hole solution is specified by four scaling-invariant parameters, namely, the dimensionless Hawking temperature $\hat{T} / \mu$, the lattice amplitude $\hat{\lambda} / \mu^{3-\Delta}$ where $\hat{\lambda} \equiv \phi(0)$, the wave vector $\hat{k} / \mu$ and $b_{0}$. For convenience, we abbreviate these quantities to $T, \lambda$ and $k$, respectively. The periodic structure with lattice constant $l \equiv 2 \pi / k$ is manifest due to the presence of the complex scalar field $\Phi$, which plays a key role in visualizing the Mott thought experiment by holography [17].

The electrical conductivity $\sigma(\omega)$ in response to the $B$ field is obtained with the standard time-dependent perturbation method in holographic setup. We adopt the following selfconsistent perturbations to the background,

$$
\delta A_{x}=a_{x}(z) e^{-i \omega t}, \delta B_{x}=b_{x}(z) e^{-i \omega t}, \delta g_{t x}=h_{t x}(z) e^{-i \omega t}, \delta \Phi=i e^{i k x} z^{3-\Delta} \varphi(z) e^{-i \omega t} .
$$

Once the background solution is obtained, the corresponding linearized perturbation equations with variables $\left(a_{x}(z), b_{x}(z), h_{t x}(z), \varphi(z)\right)$ can be solved numerically. We remark that both of gauge fields $A$ and $B$ need to be perturbed for self-consistency. Since we are especially interested in the transport properties of the Maxwell field $B$, we turn on the source $b_{x}(0)$ on the boundary but keep $a_{x}(0)=0$. We also use the diffeomorphism and gauge transformation to ensure that we are extracting the current-current correlator, which leads to an additional boundary condition $\varphi(0)-i k \lambda h_{t x}(0) / \omega=0$ on the boundary [11]. On the horizon side, the ingoing boundary conditions are imposed. When the perturbation solutions are obtained the electrical conductivity along $x$-direction can be obtained by

$$
\sigma(\omega)=\left.\frac{\partial_{z} b_{x}(z)}{i \omega b_{x}(z)}\right|_{z=0} .
$$

First of all, we intend to demonstrate the phase diagram of the system when the coupling term $\beta$ is turned on. In the case of $\beta=0$, it has been known that metal-insulator transition can occur by adjusting either of parameters $\lambda$ and $k$. However, a hard gap, which is one of the important characteristics of most Mott insulator, is absent in the optical conductivity in this original setup. In our previous work [17], we improve this by introducing the coupling term $\beta$ such that a hard gap can be manifestly observed as $\beta$ becomes large. However, in zero temperature limit an insulating phase with hard gap is never observed, which is attributed to the doped effects from the background. Instead, the system always exhibits a metallic behavior in zero temperature limit whenever a hard 
gap emerges with the increase of $\beta$. Now we intend to investigate this issue again in the context of two-gauge formalism, focusing on the transport properties of the dual system in zero temperature limit. Without loss of generality we fix $\lambda=2$ and $k=0.03$ which corresponds to an insulating phase for all values of $b_{0}$ when $\beta=0$. We plot the phase diagram over $(T, \beta)$ plane for different values of $b_{0}$, as illustrated in figure 1 . We remark that in this figure the insulating phase is determined by $\sigma_{D C}^{\prime}(T)>0$ whereas the metallic phase by $\sigma_{D C}^{\prime}(T)<0$, where the prime denotes the derivative with respect to $T$, and DC conductivity is calculated by the horizon data of the background fields [12, 17, 25]

$$
\sigma_{D C}=\left.\sqrt{\frac{V_{2}}{V_{1}}}\left[Z(\Phi)+\frac{V_{1}}{2}\left(\frac{Z(\Phi) \mu b}{k \phi}\right)^{2}\right]\right|_{z=1} .
$$

Equivalently, DC conductivity can be obtained by the zero frequency limit of AC conductivity from eq. (2.5), which has been confirmed to be identical in our numerics.

A substantial progress shown in this phase diagram is that there exists an obvious interval labelled by insulating phase between the region of metallic phase and the region of no-solution in zero temperature limit, as shown in the first two diagrams with $b_{0}=1.0$ and $b_{0}=0.8$ in figure 1 . Moreover, as we disclosed in [17], a hard gap always emerges when $\beta$ is relatively large. Above observations indicate that an insulating phase with hard gap could be achieved at zero temperature in our current system. More surprisingly, when we decrease the value of $b_{0}$, the region labelled by metallic phase shrinks and eventually disappears, as shown in the last two diagrams with $b_{0}=0.5$ and $b_{0}=0.2$ in figure 1 , which means that the system will always exhibit insulating behavior at zero temperature for arbitrary $\beta$. In [17] we propose the emergence of metallic phase is attributed to the doped property of the model, which originates from the interaction term between Maxwell field and the lattice. In our current paper it seems that the presence of additional gauge field $A$ plays a role in suppressing this doped effect. Phenomenologically, the magnitude of this suppression seems to be depicted by the ratio of chemical potential of gauge field $A$ to that of $B$, i.e. $1 / b_{0}$, which can be observed from figure 1 since the region of metallic phase shrinks with the decrease of $b_{0}$. Nevertheless, the nature of this suppression asks for further understanding.

Next we turn to demonstrate the phase diagram over $(k, \beta)$ plane with other parameters fixed. An example is given in figure 2, manifestly showing that the system undergoes a transition from a metallic phase to an insulating phase with the decrease of the wave number $k$, which is just the realization of the thought experiment proposed by Mott [5]. It is remarkable that holography can provide us such a powerful tool to visualize this scenario, as we pointed out in previous work [17]. In comparison with the phase diagram presented in [17], one key difference in our current work is that with appropriate value of $b_{0}$ (here we set $b_{0}=0.5$ ), the phase diagram over $(k, \beta)$ plane will maintain its structure down to extremely low temperature with little change. That is to say, for small values of $k$ the system will always stay in an insulating phase even in zero temperature limit, which coincides with our previous results as illustrated in figure 1. In contrast, the structure of phase diagram presented in [17] depends on the temperature evidently, where we only show an example at normal temperature. 

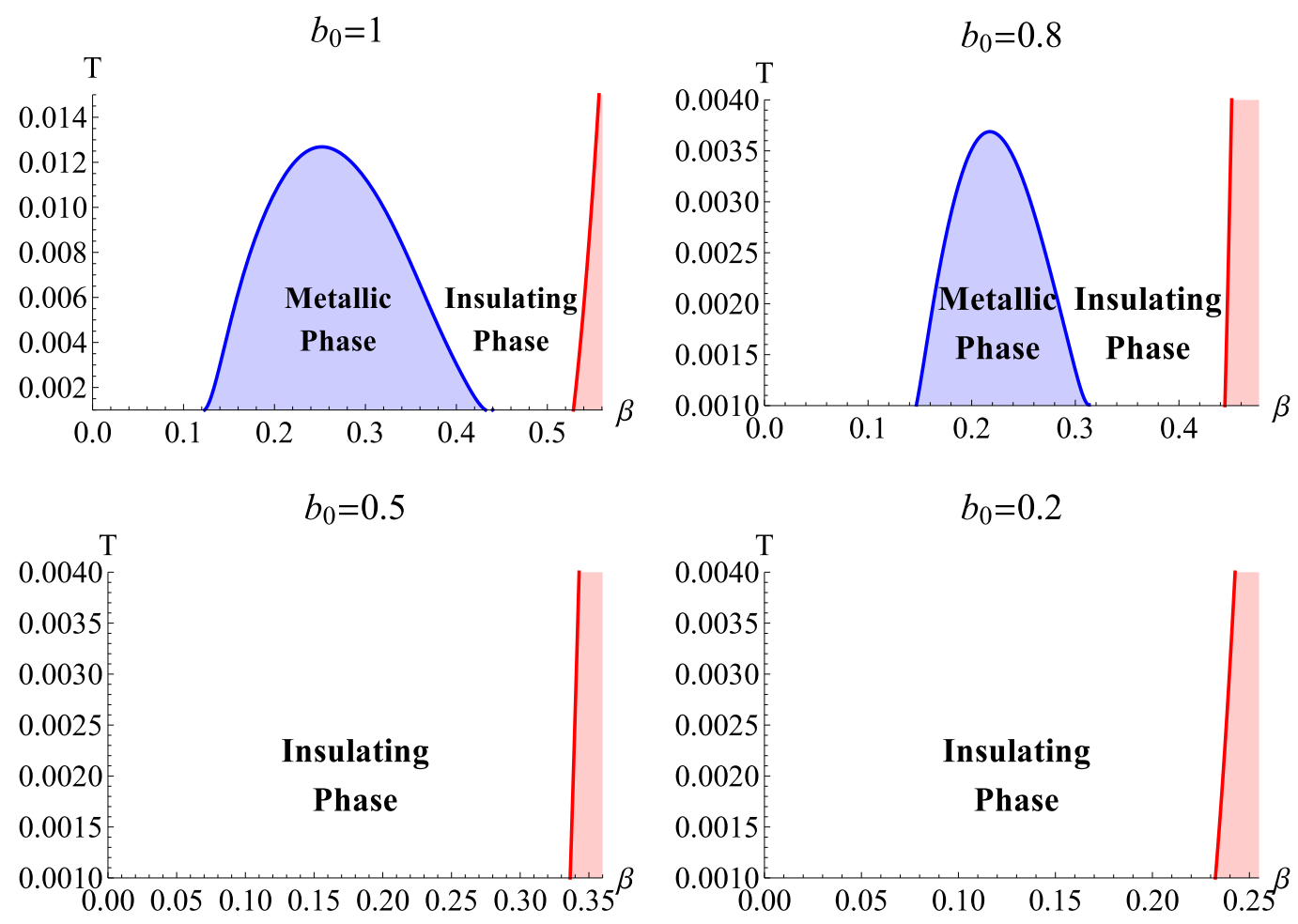

Figure 1. Phase diagrams with $b_{0}=1,0.8,0.5,0.2$ respectively from the top left to the right down. It is obviously seen that as $b_{0}$ is decreased the metallic phase region downsizes and eventually vanishes. The insulating and metallic phases are marked on each plot, the red zone is the no-solution region, in which the solution to background equations does not exist and numerically the borderline of this region is tied to vanishing charge density $\rho \simeq 0$. All the data are collected above an extremely low temperature $T / \mu=0.001$.

Another key and interesting characteristic of our dual model is the presence of a hard gap, which is one of the important properties of most Mott insulators. We demonstrate it in figure 3 by computing the frequency dependence of the optical conductivity. It is noticed that a hard gap emerges and becomes pronounced when $\beta$ becomes relatively large. We remark that all above results indicate that the novel insulating phase emerged at large $\beta$ shares some important characteristic of a Mott insulator. At phenomenological level, $\beta$ plays the same role of electron-electron interaction $U$ in Mott-Hubbard model, as has been revealed in [17]. It is also observed that the charge density $\rho$ associated with the Maxwell field $B$ is monotonously decreasing with the parameter $\beta$, and the borderline of the nosolution region is characterized by $\rho \simeq 0$. The process of $\beta$ approaching the borderline of no-solution region can be understood as the process of $U \rightarrow \infty$ where all electrons are localized.

Next we turn our interests to the properties of the dual system in zero temperature limit. First, as we have disclosed in the phase diagram, when the coupling parameter $\beta$ is large, the system lies in an insulating phase down to an extremely low temperature $\left(T \sim 10^{-5}\right)$, which is in contrast to what we observed in [17]. Second, we are concerned 


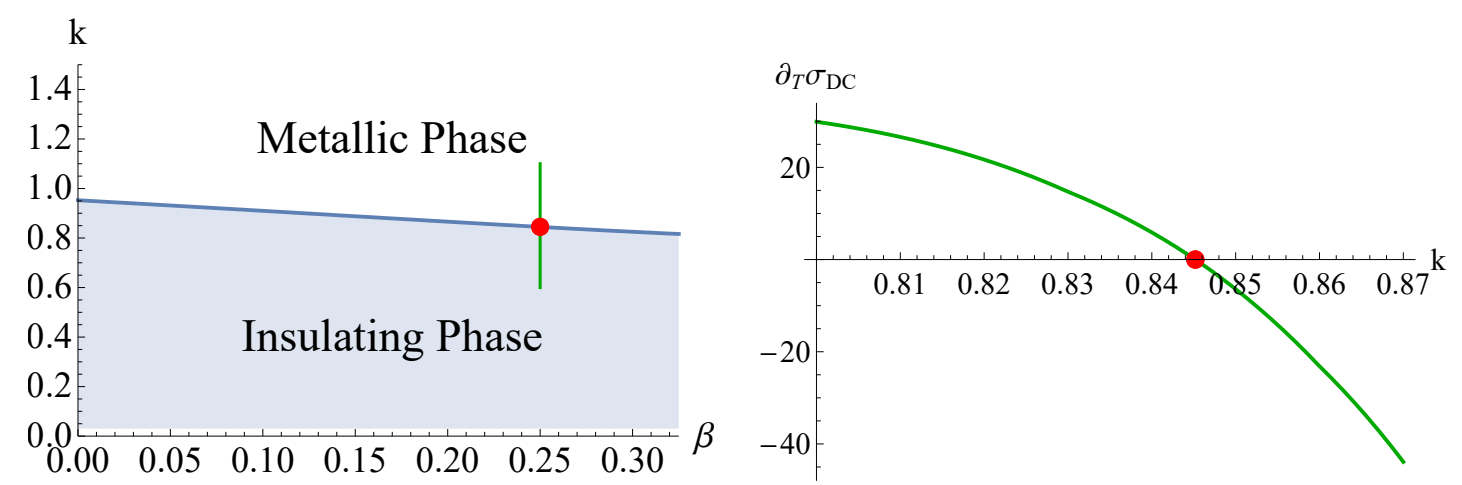

Figure 2. The left plot is the phase diagram with $\left(\lambda=2, b_{0}=0.5, T=0.001\right)$, our numerical data are collected beyond $k=0.03$. The right plot is the result of $\partial_{T} \sigma_{D C}$ v.s. $k$ at $\beta=0.25$ and other parameters are the same as the left plot. The curve in right plot corresponds to the segment of the green vertical line in the left plot, with a critical point labelled by a red dot.
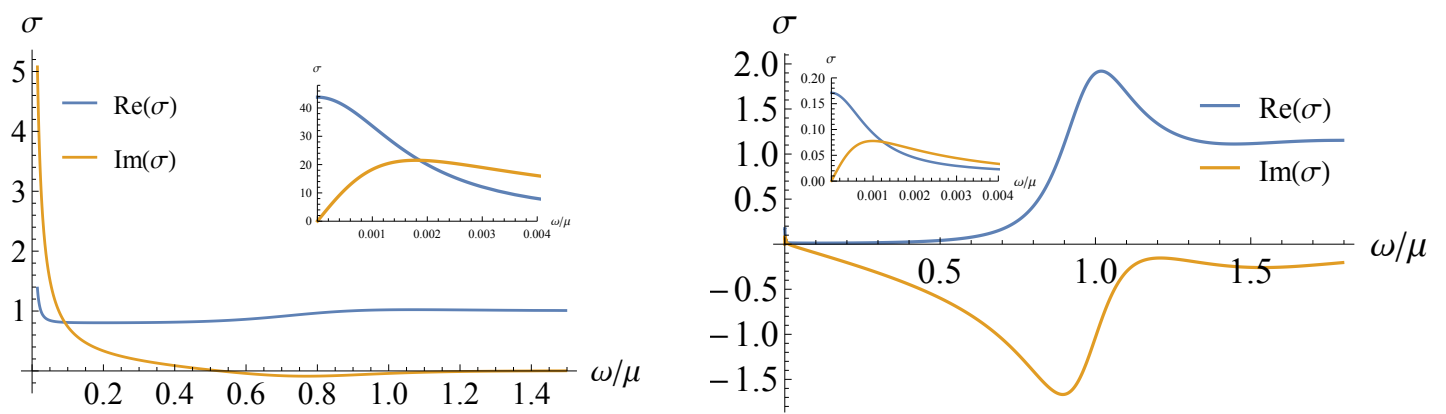

Figure 3. The optical conductivity with different values of $\beta$ (left plot is for $\beta=0$ and right one for $\beta=0.34$ ) at $T=0.005$. The other parameters are fixed as $\lambda=2, k=0.03$, and $b_{0}=0.5$. The insets in both plots are the blow-up of the optical conductivity in low frequency region.
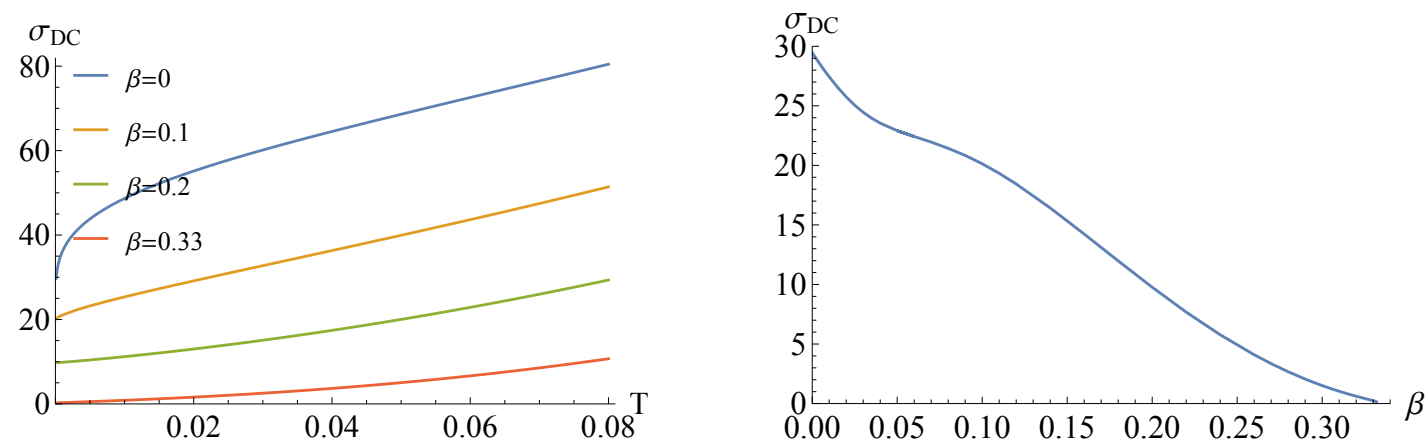

Figure 4. Left plot: DC conductivity as the function of temperature $T$ with different $\beta$. Right plot: $\mathrm{DC}$ conductivity as the function of $\beta$ with fixed temperature $T=0.0001$. All the other parameters are fixed as $\lambda=2, k=0.03$ and $b_{0}=0.5$. 


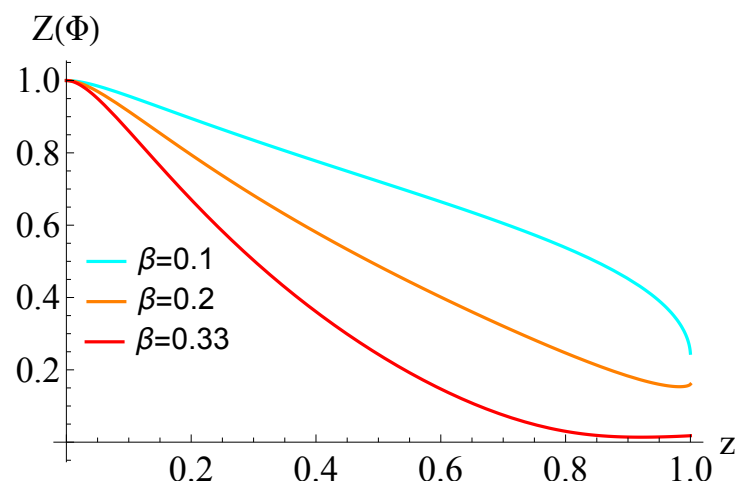

Figure 5. The coupling $Z(\Phi)$ as the function of radial coordinate $z$ with different $\beta$ at low temperature $T=0.0001$. All the other parameters are fixed as $\lambda=2, k=0.03$ and $b_{0}=0.5$.

with the behavior of the DC conductivity in zero temperature limit. From the left plot of figure 4 , when the parameter $\beta$ is small the DC conductivity may approach finite value in zero temperature limit, and the non-vanishing value decreases with the increase of $\beta$. When $\beta$ approaches the borderline of no-solution region, we find that the DC conductivity tends to vanish as the temperature goes to zero.

In holographic setup it has been understood through the near horizon analysis that to achieve a vanishing DC conductivity in zero temperature limit, one essential condition is that the coupling term $Z(\Phi)$ must be vanishing at horizon as temperature goes to zero, otherwise this term would contribute non-zero DC conductivity with both coherent and incoherent parts. Figure 5 shows the behavior of the coupling term $Z(\Phi)$ as the function of radial coordinate $z$ with different $\beta$ at extremely low temperature $T=0.0001$. We find that when $\beta$ approaches the borderline of no-solution region, the near horizon value of $Z(\Phi)$ tends to zero indeed, implying that DC conductivity also tends to zero in light of "membrane paradigm" of black holes [26] (also see eq. (2.6)).

Therefore, we conclude that we have numerically obtained a novel insulating phase with hard gap and vanishing DC conductivity in zero temperature limit, and expect it to point to an insulating ground state of the system at zero temperature. Nevertheless, we need to point out with caution that our numerical analysis above does not guarantee that some new physics might not be encountered at some ultra low temperature, as also discussed in the context of Q-lattice setup [11, 27]. We expect an analytical treatment to this system could help us to explore the solidity of this insulating ground state at absolute zero temperature in future.

In the end of this section we briefly address the issue on the zero frequency modes of optical conductivity, which exhibits some novel characteristics different from ordinary Mott-insulator. ${ }^{1}$ These modes appear simply because we are observing the system at nonzero temperature. One example is shown in figure 6 . It can be found that the conductivity in low frequency region exhibits a non-Drude behavior in an insulating state. The red

\footnotetext{
${ }^{1}$ Recent investigation on the nonvanishing Drude weight of a Mott insulator can be found, for instance in $[28,29]$.
} 

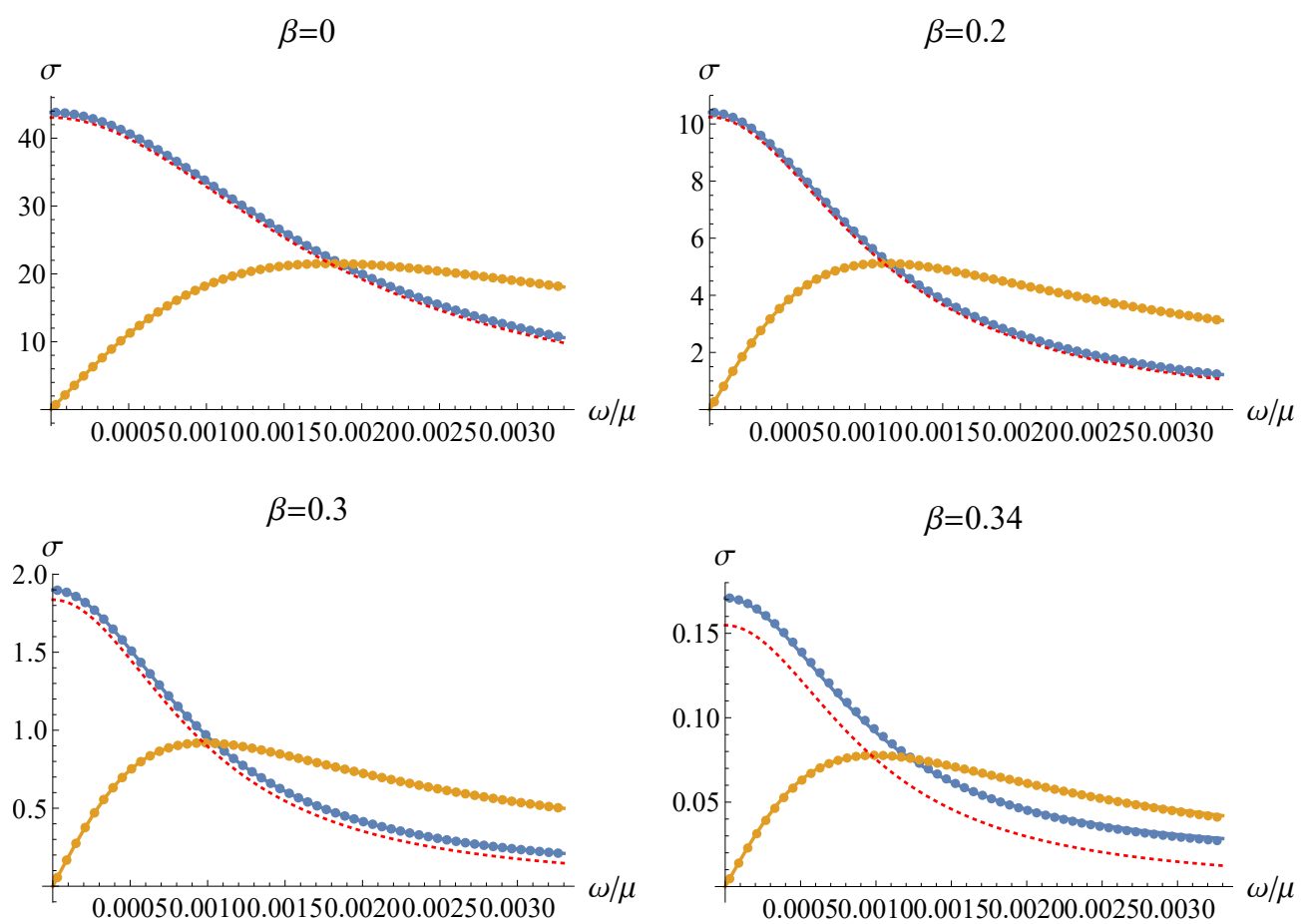

Figure 6. The low frequency behavior of the optical conductivity with $\beta=0,0.2,0.3,0.34$ respectively from the top left to right down. All the other parameters are fixed as $\lambda=2, k=0.03$ $T=0.005$ and $b_{0}=0.5$. The red dotted line in each plot is the real part of $\sigma(\omega)$ when fitted with the standard Drude formula.

dotted line in figure 6 is a fit with standard Drude formula. The deviation from the Drude law becomes evident with the increase of $\beta$. In holographic framework, such a discrepancy can be understood as the incoherent contribution which is decoupled from the momentum relaxation. In particular, stimulated by recent progress on the incoherence of conductivity [30-33], we find our data can be fitted by a modified Drude formula

$$
\sigma(\omega)=\frac{K \tau}{1-i \omega \tau}+\sigma_{Q}
$$

with constant $K$, relaxation time $\tau$ and an additional constant $\sigma_{Q} \cdot \sigma_{Q}$ comes from the incoherent contribution, which is fitted in table 1. From both figure 6 and table 1, we notice that for small $\beta, \sigma_{Q}$ is tiny compared with the coherent part such that its contribution to the total conductivity can be ignored. With the increase of $\beta$, both coherent and incoherent part are suppressed, but finally these two parts are comparable to each other such that the deviation from Drude law becomes evident. The suppression of both parts due to the presence of $\beta$ term also provides us an understanding on the emergence of hard gap and vanishing DC conductivity in our model.

\section{Discussion}

In this paper we have constructed a Q-lattice model with two U(1) gauge fields in holographic approach. After introducing a coupling term to describe the interaction between 


\begin{tabular}{|c|c|c|c|c|c|}
\hline$\beta$ & 0 & 0.1 & 0.2 & 0.3 & 0.34 \\
\hline$\sigma_{Q}$ & 0.77006 & 0.31445 & 0.16622 & 0.06259 & 0.01604 \\
\hline
\end{tabular}

Table 1. $\sigma_{Q}$ for different $\beta$ at temperature $T=0.005$. All the other parameters are fixed as $\lambda=2$, $k=0.03$ and $b_{0}=0.5$.

the lattice and the Maxwell field, we have demonstrated that the dual system is a novel insulating phase, which also resembles some important features of a Mott insulator phenomenologically. Explicitly, the system undergoes a transition from a metallic phase to insulating phase as the lattice constant becomes large, which visualizes the thought experiment proposed by Mott. A hard gap is manifestly observed as the coupling parameter becomes large. More importantly, in comparison with our previous work in [17], we have made substantial progress towards the holographic construction of Mott insulator. We have found the system stays in an insulating phase with vanishing DC conductivity in zero temperature limit, implying that the dual system points to a novel ground state which is insulating rather than a metallic phase in a doped system as described in [17]. In optical conductivity the contribution from incoherent part becomes evident as the system points to an insulating phase in zero temperature limit. We find this part could be fitted well with a modified Drude formula which contains an additional constant.

Although we have implemented some important characteristics of Mott insulator in our novel holographic insulator model, more investigations are needed to construct a holographic model dual to a realistic Mott insulator. For instance, the commensurate nature, a key ingredient of Mott insulator, has not been addressed. In addition, an analytical analysis to near horizon geometry is expected to be implemented, which could provide us more transparent understanding on the transport properties of the system in zero temperature limit.

It is completely plausible to generalize the construction in our current simple model to more complicated cases, for instance, taking the interactions between two gauge fields into account, or including a charged scalar field [34] to study the cuprate phase diagram [35, 36].

\section{Acknowledgments}

We are very grateful to Chao Niu for his collaboration during the early stage of this work. We also thank the editor and the anonymous referees for their helpful comments and suggestions. This work is supported by the Natural Science Foundation of China under Grant Nos.11275208, 11305018 and 11178002. Y.L. also acknowledges the support from Jiangxi young scientists (JingGang Star) program and 555 talent project of Jiangxi Province. J. P. Wu is also supported by the Program for Liaoning Excellent Talents in University (No. LJQ2014123).

Open Access. This article is distributed under the terms of the Creative Commons Attribution License (CC-BY 4.0), which permits any use, distribution and reproduction in any medium, provided the original author(s) and source are credited. 


\section{References}

[1] N.F. Mott, Metal-insulator transitions, Taylor and Francis, London U.K. (1974).

[2] M. Imada, A. Fujimori and Y. Tokura, Metal-insulator transitions, Rev. Mod. Phys. 70 (1998) 1039 [INSPIRE].

[3] V. Dobrosavljevic, Introduction to metal-insulator transitions, arXiv:1112.6166.

[4] D.N. Basov et al., Electrodynamics of correlated electron materials, Rev. Mod. Phys. 83 (2011) 471 [arXiv:1106.2309].

[5] N.F. Mott, The basis of the theory of electron metals, with special reference to the transition metals, Proc. Phys. Soc. London, Ser. A 49 (1937) 72.

[6] J.M. Maldacena, The large-N limit of superconformal field theories and supergravity, Int. J. Theor. Phys. 38 (1999) 1113 [hep-th/9711200] [INSPIRE].

[7] S.S. Gubser, I.R. Klebanov and A.M. Polyakov, Gauge theory correlators from noncritical string theory, Phys. Lett. B 428 (1998) 105 [hep-th/9802109] [INSPIRE].

[8] E. Witten, Anti-de Sitter space and holography, Adv. Theor. Math. Phys. 2 (1998) 253 [hep-th/9802150] [INSPIRE].

[9] A. Donos and S.A. Hartnoll, Interaction-driven localization in holography, Nature Phys. 9 (2013) 649 [arXiv:1212.2998] [INSPIRE].

[10] A. Donos, B. Goutéraux and E. Kiritsis, Holographic metals and insulators with helical symmetry, JHEP 09 (2014) 038 [arXiv:1406.6351] [INSPIRE].

[11] A. Donos and J.P. Gauntlett, Holographic Q-lattices, JHEP 04 (2014) 040 [arXiv: 1311.3292] [INSPIRE].

[12] A. Donos and J.P. Gauntlett, Novel metals and insulators from holography, JHEP 06 (2014) 007 [arXiv: 1401.5077] [INSPIRE].

[13] Y. Ling, C. Niu, J. Wu, Z. Xian and H.-b. Zhang, Metal-insulator transition by holographic charge density waves, Phys. Rev. Lett. 113 (2014) 091602 [arXiv:1404.0777] [INSPIRE].

[14] B. Goutéraux, Charge transport in holography with momentum dissipation, JHEP 04 (2014) 181 [arXiv: 1401.5436] [INSPIRE].

[15] M. Baggioli and O. Pujolàs, Electron-phonon interactions, metal-insulator transitions and holographic massive gravity, Phys. Rev. Lett. 114 (2015) 251602 [arXiv:1411.1003] [INSPIRE].

[16] E. Kiritsis and J. Ren, On holographic insulators and supersolids, JHEP 09 (2015) 168 [arXiv: 1503.03481] [INSPIRE].

[17] Y. Ling, P. Liu, C. Niu and J.-P. Wu, Building a doped Mott system by holography, Phys. Rev. D 92 (2015) 086003 [arXiv:1507.02514] [INSPIRE].

[18] A. Donos, J.P. Gauntlett and C. Pantelidou, Semi-local quantum criticality in string/M-theory, JHEP 03 (2013) 103 [arXiv: 1212.1462] [INSPIRE].

[19] A. Donos and J.P. Gauntlett, Holographic charge density waves, Phys. Rev. D 87 (2013) 126008 [arXiv: 1303.4398] [INSPIRE].

[20] M. Cvetič et al., Embedding AdS black holes in ten-dimensions and eleven-dimensions, Nucl. Phys. B 558 (1999) 96 [hep-th/9903214] [INSPIRE]. 
[21] C. Charmousis, B. Gouteraux, B.S. Kim, E. Kiritsis and R. Meyer, Effective holographic theories for low-temperature condensed matter systems, JHEP 11 (2010) 151 [arXiv: 1005.4690] [INSPIRE].

[22] B. Gouteraux and E. Kiritsis, Generalized holographic quantum criticality at finite density, JHEP 12 (2011) 036 [arXiv:1107.2116] [INSPIRE].

[23] B. Gouteraux and E. Kiritsis, Quantum critical lines in holographic phases with (un)broken symmetry, JHEP 04 (2013) 053 [arXiv:1212.2625] [INSPIRE].

[24] E. Mefford and G.T. Horowitz, Simple holographic insulator, Phys. Rev. D 90 (2014) 084042 [arXiv: 1406.4188] [INSPIRE].

[25] Y. Ling, P. Liu, C. Niu, J.-P. Wu and Z.-Y. Xian, Holographic entanglement entropy close to quantum phase transitions, arXiv:1502.03661 [INSPIRE].

[26] N. Iqbal and H. Liu, Universality of the hydrodynamic limit in AdS/CFT and the membrane paradigm, Phys. Rev. D 79 (2009) 025023 [arXiv:0809.3808] [INSPIRE].

[27] A. Donos and J.P. Gauntlett, Superfluid black branes in $A d S_{4} \times S^{7}$, JHEP 06 (2011) 053 [arXiv: 1104.4478] [INSPIRE].

[28] S. Fujimoto and N. Kawakami, Exact Drude weight for the one-dimensional Hubbard model at finite temperatures, J. Phys. A 31 (1998) 465.

[29] H. Nakano, Y. Takahashi and M. Imada, Drude weight of the two-dimensional Hubbard model - Reexamination of finite-size effect in exact diagonalization study, J. Phys. Soc. Jpn. 76 (2007) 034705.

[30] K.-Y. Kim, K.K. Kim, Y. Seo and S.-J. Sin, Coherent/incoherent metal transition in a holographic model, JHEP 12 (2014) 170 [arXiv:1409.8346] [INSPIRE].

[31] X.-H. Ge, Y. Ling, C. Niu and S.-J. Sin, Thermoelectric conductivities, shear viscosity and stability in an anisotropic linear axion model, Phys. Rev. D 92 (2015) 106005 [arXiv: 1412.8346] [INSPIRE].

[32] R.A. Davison, B. Goutéraux and S.A. Hartnoll, Incoherent transport in clean quantum critical metals, JHEP 10 (2015) 112 [arXiv:1507.07137] [INSPIRE].

[33] R.A. Davison and B. Goutéraux, Dissecting holographic conductivities, JHEP 09 (2015) 090 [arXiv: 1505.05092] [INSPIRE].

[34] Y. Ling, P. Liu, C. Niu, J.-P. Wu and Z.-Y. Xian, Holographic superconductor on Q-lattice, JHEP 02 (2015) 059 [arXiv: 1410.6761] [INSPIRE].

[35] E. Kiritsis and L. Li, Holographic competition of phases and superconductivity, JHEP 01 (2016) 147 [arXiv: 1510.00020] [INSPIRE].

[36] M. Baggioli and M. Goykhman, Under the dome: doped holographic superconductors with broken translational symmetry, JHEP 01 (2016) 011 [arXiv:1510.06363] [INSPIRE]. 